\title{
Processing Coordinated Structures in Context: The Effect of Topic-Structure on Ambiguity Resolution
}

\author{
John C. J. Hoeks \\ Department of Linguistics, University of Nijmegen, The Netherlands \\ Wietske Vonk \\ Max Planck Institute for Psycholinguistics, Nijmegen, and Department of Linguistics, \\ University of Nijmegen, The Netherlands
}

and

Herbert Schriefers

Nijmegen Institute for Cognition and Information, University of Nijmegen, The Netherlands

\begin{abstract}
When a sentence such as The model embraced the designer and the photographer laughed is read, the noun phrase the photographer is temporarily ambiguous: It can be either one of the objects of embraced (NP-coordination) or the subject of a new, conjoined sentence (S-coordination). It has been shown for a number of languages, including Dutch (the language used in this study), that readers prefer NP-coordination over S-coordination, at least in isolated sentences. In the present paper, it will be suggested that NP-coordination is preferred because it is the simpler of the two options in terms of topic-structure; in NP-coordinations there is only one topic, whereas S-coordinations contain two. Results from off-line (sentence completion) and online studies (a self-paced reading and an eye tracking experiment) support this topic-structure explanation. The processing difficulty associated with S-coordinated sentences disappeared when these sentences followed contexts favoring a two-topic continuation. This finding establishes topic-structure as an important factor in online sentence processing. (c) 2001 Elsevier Science
\end{abstract}

Key Words: sentence processing; context; pragmatics; topic-structure; coordination; eye tracking.

A considerable amount of research has been dedicated to finding out how information from extrasentential context can affect the syntactic processing of a subsequent sentence. In an influential article, Crain and Steedman (1985) drew a first sketch of how contextual information might be taken into account by the parser. For example, their principle of referential success stipulates that "if there is a reading that succeeds in referring to an entity already established in the

We would especially like to thank Laurie Stowe for helpful comments on an earlier version of this paper. The complete set of Dutch materials used in the experiments can be obtained in electronic form from http://www.idealibrary.com.

Address correspondence and reprint requests to John C. J. Hoeks, Department of Linguistics, University of Groningen, P.O. Box 716, 9700 AS Groningen, The Netherlands. E-mail: hoeks@let.rug.nl. hearer's mental model of the domain of discourse, then it is favored over one that does not" (p. 331). To illustrate, when presented with a sentence such as 1, readers will experience processing difficulty at to visit him again, because they preferentially take the that-clause as a complement, instead of as a relative clause (e.g., Altmann, Garnham, \& Dennis, 1992; Crain \& Steedman, 1985).

The psychologist told the woman that he was having trouble with to visit him again.

Crain and Steedman suggested that this preference arises because such a relative clause is generally used to restrict a set of possible referents (e.g., in cases where there is more than one woman present in the discourse context). For sentences in isolation, as well as for contexts in which there is no referential ambiguity, the use 
of a relative clause is rather unexpected, causing the that-clause to be read as a complement clause instead. However, if the discourse context were to contain two (or more) equally plausible referents for the woman, the preference would switch in favor of the relative clause reading.

Although Crain and Steedman's model is often referred to as a "referential" theory, and a fair number of studies have been conducted to test the principle of referential success (e.g., Altmann \& Steedman, 1988; van Berkum, Brown, \& Hagoort, 1999; Britt, 1994; Clifton \& Ferreira, 1989; Mitchell, Corley, \& Garnham, 1992; Murray \& Liversedge, 1994; Ni, Crain, \& Shankweiler, 1996; Rayner, Garrod, \& Perfetti, 1992; Spivey \& Tanenhaus, 1998; Zagar, Pynte, \& Rativeau, 1997), the scope of their theory clearly goes beyond referential processing per se. The principle of referential success is, in fact, merely a special case of the principle of parsimony, in which it is stated that, in case of syntactic ambiguity, the alternative that requires fewest accommodations will be chosen, with accommodations being changes to a model of the discourse.

In the present paper, a principle called the principle of minimal topic-structure will be proposed, that can be viewed as another instance of the principle of parsimony. It will be argued that this new principle is central to the resolution of a certain kind of structural ambiguity, namely, the NP- versus S-coordination ambiguity. This ambiguity arises in structures such as (2a), where the photographer can be either part of the direct object of embraced, as in (2b) (NP-coordination), or the subject of a conjoined sentence, as in (2c) (S-coordination).

\footnotetext{
The model embraced the designer and the photographer.
}

The model embraced [the designer and the photographer] at the party.

[The model embraced the designer] and [the photographer laughed].

Previous research (e.g., Frazier, 1987a) has shown that readers are initially inclined to interpret the ambiguous NP as part of a complex object, which causes them to incur processing difficulty in S-coordinated sentences such as (2c). It has been argued that this conjoint-NP preference reflects the workings of a processor that chooses the simplest alternative in terms of syntactic structure. For instance, in the framework of garden-path/construal theory, a phrasemarker describing NP-coordination requires the fewest nodes and will hence be chosen by application of the minimal attachment strategy (Frazier, 1987b; Frazier \& Clifton, 1996, 1997). Other theories, such as the constraint-based models, would explain the NP-coordination preference as the outcome of a constraint-satisfaction process, in which different factors may provide different degrees of support for one or the other alternative (MacDonald, Pearlmutter, \& Seidenberg, 1994; Tanenhaus \& Trueswell, 1995). In the case of the NP versus S-coordination ambiguity, the most likely candidate to play a role in this process is the frequency of the different structures involving the connective. In a corpus consisting of one edition of the Dutch daily newspaper Trouw, 61\% of all occurrences of en ('and') that were analyzed ( $\mathrm{n}=$ 406) were NP-coordinations, $16 \%$ were VPcoordinations, and $10 \%$ S-coordinations. However, if we look at contingent frequencies, that is, frequencies that take grammatical function into account, this picture changes quite radically. Coordinated NPs functioning as grammatical objects occur only $6 \%$ of the time; Scoordinations with two different subjects make up for a mere $7 \%$ of all cases, while VP-coordinations are most frequent with $16 \%$. So depending on the frequency measure used (i.e., coarse-grained or more fine-grained), constraint-based models will predict either a strong NP-coordination preference or a preference for VP-coordination, respectively. However, in this paper, it will be proposed that the NP-coordination preference is not primarily motivated by syntactic simplicity or lexical frequency of 'and', but that it arises because readers choose the alternative that is the least complex in terms of its topic-structure.

Topic-structure can be loosely defined as describing the relation between the topic of a sentence, i.e., the element referring to an entity about which information is given, and the "new" 
information that is expressed by a sentence, or comment. The most common topic-structure is the so-called topic-comment structure which is typical for the vast majority of sentences in discourse (see Lambrecht, 1994, p. 132, for discussion and references). This structure is characterized by a subject serving as the topic, followed by the comment, a predicate which "contains" the new information about the subject, such as in The woman walked to the car, where the woman is topic and walked to the car is the comment. Other topic-structures are the identificational structure, in which the "new" element is not the predicate but an argument (e.g., "It was the woman who walked to the car,' where the information is presented that it was the woman and not, e.g., the man who walked to the car), and the presentational structure, in which the entire sentence consists of new information (e.g., 'A woman walked to a car,' where the whole event is presented as a piece of new information).

What we would like to propose is that, in the course of reading, readers construct a topicstructure representation of the sentence at hand. If a sentence appears discourse-initial or in isolation, and if there are no explicit syntactic cues regarding what is topic and what is not, the reader will assume some kind of default, minimal topic-structure. This hypothesis can be formulated into the principle of minimal topicstructure, as given in (3).

\begin{abstract}
Principle of minimal topic-structure: In the absence of explicit contextual or syntactic cues regarding the topic-structure of a sentence, assume the simplest topic-structure possible.
\end{abstract}

The most likely candidate for this minimal topic-structure is the topic-comment structure, which is characteristic for most sentences in discourse (Lambrecht, 1994). This seems to be a plausible assumption, since it is surely more common for a sentence to convey information about given discourse entities than for it to introduce new entities into the discourse, or to report events out of the blue (Lambrecht, 1994). More importantly, besides being the most frequently used topic-structure, topic-comment structure can also be considered the least complex topic-structure in terms of the mental effort needed in accommodating new referents or new information: It only requires accommodating one topic, about which information is then added. The identificational structure, on the other hand, requires the accommodation of both the topic and a certain set of other referents the given topic is contrasted with. Furthermore, we would like to speculate that topic-comment structure is also less complex than the presentational structure, which is typically used to start a whole new story or a separate subpart of the main story (Lambrecht, 1994). It seems reasonable to assume that processing new information and creating a new (part of a) discourse model require extra mental effort.

In conclusion, then, topic-comment structure seems to be the most plausible default, minimal topic-structure. Consider, for example, the temporarily ambiguous sentence $(2 \mathrm{c})$, repeated here for convenience as (4).

The model embraced the designer and the photographer laughed.

For the writer of this sentence it is, of course, absolutely clear that the sentence contains two topics, the model and the photographer, who each perform a distinct action. Readers, however, do not have this information, and only when the last word (laughed) is read will they know that this sentence is not a standard topic-comment sentence with the model as its (sole) topic. Instead, when they reach the disambiguating verb laughed, they will find out that the photographer is not part of the comment as they might have assumed but that it is a topic in its own right, which means that they will have to accommodate the photographer as a second topic in the sentence. This led us to the following hypothesis about the NP-coordination preference in sentences such as (4): Readers do not prefer NP-coordination because of syntactic simplicity, or because of the connective and being used more often in NP-coordinations, but they disprefer S-coordination when this involves having to complicate the assumed topic-comment structure by having to accommodate the ambiguous NP as a second topic.

If this explanation is true, then it should in principle be possible to eliminate the processing difficulty in ambiguous S-coordinated sentences if we can devise contexts that change the ex- 
pected topic-structure of such sentences. Consider the question in (5).

What did the model and the photographer do?

This question establishes both the model and the photographer as discourse entities about which something is going to be said: Both will be very likely to become topics of the following sentence. Note that, initially, the reader could assume that the two entities together form a "duplex topic," meaning that both might be taken to perform some action in concord. Indeed, a very natural answer to the question in (5) would be something like 'They drank a glass of champagne'. However, when a subsequent sentence starts out with 'The model . . .' (instead of with 'They .. .'), this might inform the reader that the actions of the entities involved will each be discussed in their own right (i.e., as contrastive topics; see Lambrecht, 1994). So by manipulating the context it should be possible to guide the reader in adopting a nondefault topic-structure for an S-coordinated sentence; a revision of the assumed topic-structure is no longer necessary and hence no processing difficulty is expected.

We will present studies showing that contexts such as (5) are effective in changing the topicstructure of an upcoming sentence (Experiment 1 , gated completion) and that this change in topic-structure can eliminate the processing difficulty observed in S-coordinated sentences in isolation (Experiment 2, self-paced reading, and Experiment 3, eye tracking).

\section{EXPERIMENT 1}

Experiment 1 consisted of five fragment completion substudies. The first two substudies looked at the completion of sentence fragments in isolation, whereas the other three investigated fragment completion in contexts biasing toward either NP-coordination or S-coordination. To investigate the strength of the NP-coordination bias at different points in the sentence, a gated completion paradigm was used. Participants were presented with three kinds of sentence fragments: (1) ending with the connective, as in 'The model embraced the designer and . . .'; (2) ending with the connective plus the article, 'The model embraced the designer and the . . .; or
(3) ending with connective, article, and noun, 'The model embraced the designer and the photographer . . .' See Table 1 for an overview of the different substudies.

In the context conditions, "question-contexts" such as (6a), as well as "sentence-contexts" such as (6b) were used to bias toward S-coordinated completions.
The party at the end of the fashion show was very exciting. What did the model and the photographer do?

When they met the fashion designer at the party, the model and the photographer were very enthusiastic.

In both of these contexts, the two intended topics were presented as subject of the main clause. As to the sentential contexts, since simply mentioning two discourse entities in a sentence does not ensure that these entities will serve as topic in the next sentence, the context sentences had to be clearly "presentational," in the sense that they should place the two intended topics into the center of attention. According to Lambrecht (1994), the cataphoric use of a pronoun, such as they in the preposed subclause, will have the effect of emphasizing the importance of the entities it refers to (i.e., the model and the photographer). Such a cataphor construction belongs to the same class of presentational devices as, for example, the well-known 'Once upon a time . . ' (Lambrecht, 1994). Using the cataphor construction has the advantage of allowing for the introduction of a third important entity, the designer, so that, when the context sentence has been read, each referent in the following target-sentence will have been properly introduced. As we shall see, sentence-contexts proved to be as effective as question-contexts in changing the expected topic-structure of target-sentences.

\section{Method}

Participants. In total, 170 undergraduate students from the University of Nijmegen took part. None of the participants in this experiment, or in the other two experiments reported in this paper, had previously taken part in experiments involving coordinated structures. The number of participants in each of the five completion substudies can be found in Table 1 . 
TABLE 1

Features of the Five Completion Substudies of Experiment 1

\begin{tabular}{|c|c|c|c|c|}
\hline Context type & Substudy & Fragment ending & No. lists & No. participants \\
\hline \multirow[t]{2}{*}{ No context } & $1 \mathrm{a}$ & and . . . & 1 & 16 \\
\hline & $1 b$ & $\begin{array}{l}\text { and the ... } \\
\text { and the photographer. }\end{array}$ & 2 & 26 \\
\hline \multirow[t]{3}{*}{ Context } & $1 \mathrm{c}$ & and... & 4 & 52 \\
\hline & $1 d$ & and the ... & 4 & 44 \\
\hline & $1 \mathrm{e}$ & and the photographer. . . & 4 & 32 \\
\hline
\end{tabular}

Materials. In all substudies 16 experimental sentence fragments were used, which were essentially the same for each substudy (i.e., except for their cutoff point, see below). All materials of this and subsequent experiments were in Dutch. An example of an experimental fragment is given in 2.1 in Table 2. The slashes indicate the three points where fragments ended. In the context substudies, four contexts were constructed for each sentence fragment. See Table 2 for examples. Each of these contexts was designed to promote either a "simplex" or a "duplex" topic continuation. A simplex topic continuation would be a continuation with just one topic (i.e., the referent that is already present in the sentence fragment, the model). Such a continuation may take the form of a VP-coordination, of which (7a) is an example, or an NP-coordination, as given in (7b). A duplex topic continuation would contain two topics and would take the form of a sentence-coordination with two topics, as in (7c) (literal translations in apostrophes).

De mannequin omhelsde de ontwerper en
verliet het feest.
'The model embraced the designer and left
the party.'

De mannequin omhelsde de ontwerper en de fotograaf op het feest.

'The model embraced the designer and the photographer at the party.'

De mannequin omhelsde de ontwerper en de fotograaf lachte.

'The model embraced the designer and the photographer laughed.'

All duplex-topic sentence contexts had the form of sentence 2.4 in Table 2 and consisted of a preposed subclause followed by a main clause. The subclause always started with a connective (when, because, while, etc.) and contained a plural (cataphoric) pronoun, the NP that will become the object NP in the target-sentence, a verb, and sometimes some adverbial material. The main clause consisted of a verb-phrase starting with the main verb, followed by the NPcoordinated subject (i.e., referring to the two entities that are the intended topics of the targetsentence) and one to seven extra words. A simplex-topic sentence context contained the same words, but in a different order, as shown in sentence 2.2 in Table 2. Here, the intended topic of the next sentence, the model, is contained in the main clause, whereas the other two entities are present in the subclause. Question-contexts consisted of an introductory declarative sentence followed by a short question, in which either one (simplex-topic condition; 2.3 in Table 2) or two (duplex-topic condition; 2.5 in Table 2) entities were mentioned.

In all substudies, 22 filler fragments of varying syntactic structure were added to the experimental fragments (e.g., The sultan banned the rebel . . .). In the context substudies, all of the fillers were preceded by contexts, half of which resembled a sentence context, while the other half consisted of a declarative sentence followed by a question.

Design and procedure. In substudy 1a, 16 experimental fragments ending with 'and ...' were pseudo-randomly interspersed among 22 filler items (allowing maximally two experimental items in consecutive order). The order of items was the same for each participant. For substudy $1 \mathrm{~b}$, two lists were constructed with equal 
TABLE 2

Sample Materials Used in the Substudies of Experiment 1, with English Translations

Sample sentence fragment

2.1 De mannequin omhelsde de ontwerper en / de / fotograaf / . .

'The model embraced the designer and / the / photographer /. . .'

Sample contexts

Simplex-topic sentence context:

2.2 Toen ze de modeontwerper en de fotograaf op de party ontmoette, was de mannequin heel enthousiast.

'When she met the fashion designer and the photographer at the party, the model was very enthusiastic.'

Simplex-topic question context:

2.3 Het feest na afloop van de mode show was erg uitbundig. Wat deed de mannequin?

'The party at the end of the fashion show was very exciting. What did the model do?

Duplex-topic sentence context:

2.4 Toen ze de modeontwerper op de party ontmoetten, waren de mannequin en de fotograaf heel enthousiast.

'When they met the fashion designer at the party, the model and the photographer were very enthusiastic.'

Duplex-topic question context:

2.5 Het feest na afloop van de mode show was erg uitbundig. Wat deden de mannequin en de fotograaf?

'The party at the end of the fashion show was very exciting. What did the model and the photographer do?

Note. Slashes in the example sentence fragment indicate where the fragments ended in the different completion studies.

numbers of fragments occurring in each condition (i.e., fragments ending with 'and the. . .' and fragments ending with 'and the photographer. . .). A list contained only one version of each fragment, fragments were presented in the same order as in substudy $1 \mathrm{a}$, together with the same fillers, and each list was seen by an equal number of participants. The three context substudies $1 \mathrm{c}$ to $1 \mathrm{e}$ each targeted a different type of fragment (i.e., 'and . . .' in 1c, 'and the . . .' in $1 \mathrm{~d}$, 'and the photographer . . .' in 1e). For each of these substudies, four lists were created using a Latin Square, with equal numbers of sentences occurring in each condition (i.e., the four context conditions: simplex-topic sentence context, simplex-topic question context, duplex-topic sentence context, and duplex-topic question context). A list contained only one version of a context condition, with experimental and filler fragments appearing in the same pseudo-random order as in the previous studies. In each particular substudy, each list was seen by an equal number of participants.

Participants were instructed to read the fragments carefully and to write down the first grammatical and meaningful continuation that came to mind. At the same time it was stressed that this completion should result in a coherent and plausible sentence (no-context condition) or story (context conditions).

\section{Results and Discussion}

Continuations were assigned one of four categories, VP-coordination (e.g., The model embraced the designer and left the party), NPcoordination (e.g., The model embraced the designer and the photographer at the party), S-coordination (e.g., The model embraced the designer and the photographer laughed), and finally the category Unclassifiable, for ambiguous or ungrammatical responses. The results of Experiment 1 are presented in Table 3.

A number of important findings emerged from this completion experiment. First, clear evidence was found for the viability of the principle of minimal topic-structure. In sentence fragments presented without any context, the majority of completions were of the simplextopic kind. In the case of fragments ending with 'and .. .' (substudy 1a), this was generally accomplished by producing VP-coordinations $(86 \%)$. The reason for this preponderance of VP-coordinations might be related to the frequency with which en ('and') occurs in different syntactic structures. Recall that, in a Dutch corpus, $16 \%$ of all structures containing en were VP-coordinated, versus $6 \%$ with coordinated NPs, at least if we look at the fine-grained measures. On the other hand, VP-coordination might be preferred over NP-coordination for (pragmatic) reasons that remain to be identified. After 
TABLE 3

Percentages of Completion as VP-Coordination (2VP), NP-Coordination (2NP), or S-Coordination (2S) for Fragments Presented in Isolation and in Context in Experiment 1 (Standard Errors Given in Parentheses)

\begin{tabular}{llrrrr}
\hline \multicolumn{1}{c}{ Context type } & \multicolumn{1}{c}{ Fragment ending } & 2VP & 2NP & 2S & Substudy \\
\hline No context & and . . & $86(4)$ & $9(3)$ & $5(2)$ & $1 \mathrm{a}$ \\
& and the . . & $0(0)$ & $87(3)$ & $13(3)$ & $1 \mathrm{~b}$ \\
& and the photographer . . . & $0(0)$ & $81(3)$ & $15(2)$ & $1 \mathrm{~b}$ \\
Simplex-topic, sentence context & and . . & $74(5)$ & $19(4)$ & $3(1)$ & $1 \mathrm{c}$ \\
& and the . . & $3(2)$ & $56(8)$ & $40(8)$ & $1 \mathrm{~d}$ \\
& and the photographer . . . & $0(0)$ & $76(5)$ & $23(5)$ & $1 \mathrm{e}$ \\
Simplex-topic, question context & and . . & $89(4)$ & $8(3)$ & $0(0)$ & $1 \mathrm{c}$ \\
& and the . . & $6(2)$ & $41(6)$ & $51(6)$ & $1 \mathrm{~d}$ \\
& and the photographer. . . & $0(0)$ & $73(6)$ & $27(6)$ & $1 \mathrm{e}$ \\
Duplex-topic, sentence context & and . . & $55(6)$ & $2(1)$ & $37(8)$ & $1 \mathrm{c}$ \\
& and the . . & $0(0)$ & $15(4)$ & $76(5)$ & $1 \mathrm{~d}$ \\
& and the photographer . . . & $0(0)$ & $18(5)$ & $80(5)$ & $1 \mathrm{e}$ \\
Duplex-topic, question context & and . . & $42(6)$ & $1(3)$ & $51(6)$ & $1 \mathrm{c}$ \\
& and the . . & $2(9)$ & $11(2)$ & $77(3)$ & $1 \mathrm{~d}$ \\
& and the photographer . . . & $0(0)$ & $19(4)$ & $80(4)$ & $1 \mathrm{e}$ \\
\hline
\end{tabular}

all, there must be an explanation of why VP-coordination occurs more frequently than NP-coordination. When VP-coordination was eliminated as a possibility, however, as in the fragments ending with 'and the. . .' or 'and the photographer' (substudy 1b), NP-coordination was clearly the favored option (over $80 \%$ ).

The second important result was the effectiveness of duplex-topic contexts in promoting S-coordination completions with two topics. The largest effect of this kind of context was present in the fragments ending with 'and the photographer . . .' (substudy 1e), where $80 \%$ of the completions were S-coordinations, against a mere $19 \%$ of NP-coordinations.

Finally, simplex-topic contexts predominantly biased toward VP-coordinations in the fragments ending with 'and . . .' (substudy 1c) and toward NP-coordinations in the fragments containing the full NP (substudy 1e). In the 'and the . . .' case (substudy 1d), however, there was also a clear number of S-coordinated completions, ranging from $40 \%$ in the sentence contexts to $51 \%$ in the question contexts. It is possible that the mere presence of a great many $\mathrm{S}$-coordinations, given in response to the duplex-topic contexts of the same list, counteracted the expected bias toward NP-coordination.

In sum, sentence fragments containing and (i.e., en in the Dutch materials) are preferably completed in a way preserving the topic-comment structure with one and only one topic, that is, as VP-coordinations or as NP-coordinations, just as was predicted by the principle of minimal topic-structure. However, the results from this experiment also show that this preference can be modulated by duplex-topic contexts.

\section{EXPERIMENT 2}

The results of Experiment 1 showed that sentence and question contexts were both effective in manipulating the topic-structure of ambiguous sentence fragments. In Experiment 2 , we investigated whether this manipulation of topic-structure can eliminate the processing difficulty observed in temporarily ambiguous S-coordinations presented without a context (Frazier, 1987a; Frazier \& Clifton, 1997). Sentence (8a) shows an example of a temporarily ambiguous target sentence (the literal English translation is given in apostrophes). These sentences were compared with unambiguous (S-coordinated) control sentences such as (8b), created by adding a comma to the object NP the designer.

De mannequin omhelsde de ontwerper en de fotograaf opende lachend een fles champagne.

'The model embraced the designer and the photographer opened smilingly a bottle of champagne.' 
De mannequin omhelsde de ontwerper, en de fotograaf opende lachend een fles champagne. 'The model embraced the designer, and the photographer opened smilingly a bottle of champagne.'

It should be noted that in Dutch (the language used in the present experiments), there are no strict rules regarding the placement of a comma in S-coordinated sentences (Geerts, Haeseryn, De Rooij, \& Van den Toorn, 1984; Renkema, 1979). There is, however, a general habit of not putting commas before en ('and') in conjoined sentences, and it is definitely not acceptable to use a comma in cases where two NPs are conjoined. So the absence of a comma does not provide much useful information regarding the structure of a sentence, whereas its presence signals that S-coordination (or VP-coordination, for that matter) is very likely but NP-coordination is highly improbable. Any processing difficulty in the ambiguous sentence will thus show up as a difference in reading times when compared to the unambiguous control sentence.

For each experimental sentence, two kinds of critical context-sentences were constructed, a biasing context and a neutral context. The biasing context-sentences (i.e., biasing toward the unpreferred S-coordination reading) had the same structure as the duplex-topic sentence contexts described in Experiment 1. These biasing contexts were contrasted with neutral contexts in which none of the protagonists is mentioned, as in (9).

It was not surprising that the party after the fashion show was exhilarating.

The choice to use neutral contexts instead of the simplex-topic contexts from Experiment 1 was based on the assumption that using a neutral context would provide the strongest test of the principle of minimal topic-structure, since such a context contains no clue whatsoever as to the topic-structure of the next sentence. Readers will apply the default topic-structure only in a neutral context, while a simplex-topic context may already guide the reader toward a simplextopic reading in the next sentence. Furthermore, using neutral contexts will enable us to directly compare the results with the outcome of earlier studies, where sentences did not have a context at all (e.g., Frazier, 1987a). One potential problem is that these neutral contexts may make the target-sentences somewhat infelicitous, but in fact, these sentences are no more infelicitous than if they were presented in isolation. Furthermore, by setting a specific scene (e.g., a fashion show), the neutral contexts make the introduction of specific, scene-related referents in the target-sentence easier than if they were presented out of the blue. Nevertheless, it must be acknowledged that target-sentences following biasing contexts are more felicitous than those following neutral contexts, since the entities referred to by definite NPs (e.g., the designer and the photographer) have been mentioned in the biasing contexts but not in the neutral ones. We will come back to this issue when we discuss the results of Experiments 2 and 3.

The following predictions are made. The NPcoordination bias that was shown to affect the processing of temporarily ambiguous S-coordinations presented in isolation (Frazier, 1987a; Frazier \& Clifton, 1997) will also cause processing difficulty in sentences preceded by a neutral context, since such a context contains no information as to how to process the next sentence. Readers will, in the absence of clear cues, fall back on the strategy of applying a default, minimal topic-structure to this sentence (i.e., assume a topic-comment structure with only one topic) and will run into trouble when a disambiguating verb appears, telling them that the ambiguous NP is a topic too. There will be no processing difficulty in sentences embedded in a biasing duplex-topic context, since this context makes it clear from the beginning that the ambiguous NP will be a topic.

\section{Method}

Participants. The participants were 32 undergraduate students from the University of Nijmegen; all were paid for taking part in the experiment.

Materials. A set of 48 experimental sentences were constructed, all of which were sentencecoordinations (16 of these sentences were formed on the basis of the sentence fragments used in Experiment 1). All sentences were of the following structure: [NP-Verb-NP]-connec- 
tive-[NP-Verb-Adverb-NP]-rest of the sentence. In the control sentences a comma was added to the second NP (i.e., the object NP). The postverbal adverb in the clause following the connective was included to "focus" possible spillover effects originating from the disambiguating verb on a single content-word, instead of on a NP (i.e., article plus noun). This is relatively unimportant for the self-paced reading in Experiment 2, since the article and the noun of the postverbal NP were presented together as one segment. However, it might be important for the replication with eye tracking in Experiment 3 , since a content word is less likely to be skipped than an article, making measurement of spillover effects more straightforward. For each of the experimental sentences, biasing and neutral contexts were created, consisting of a leadin sentence, a neutral or a biasing context-sentence, the target-sentence, and an exit-sentence. For examples see Table 4.

Besides the 48 experimental sentences involving $\mathrm{S}$-coordination, the reader was also presented with 48 filler sentences which contain NP-coordinations, in order to minimize the chance that participants would develop strategies or grow accustomed to S-coordinations. For half of these filler sentences, contexts were produced in which two protagonists were explicitly mentioned, just as in the biasing context condition. Note that in "biasing" filler items, the critical context leads the reader to expect that the next sentence will contain two topics, but the subsequent filler sentence only provides one. The filler sentence is thus infelicitous in these stories. The main reason for including these stories, despite their infelicity, was to prevent participants from using strategies. For the same reason we also constructed 24 "neutral" filler stories. See Table 5 for sample filler items. There were no other fillers in this experiment. ${ }^{1}$

\footnotetext{
${ }^{1}$ No comprehension questions were used in this experiment. In an unpublished study, we tested whether adding comprehension questions would affect the reading behavior of participants, especially with respect to the size of the garden-path effect ensuing from the NP/S ambiguity in sentences presented in isolation. Two self-paced reading experiments were conducted on identical materials, where one of the experiments contained content questions, whereas the
}

The materials for the practice session consisted of one story with an NP-coordinated target sentence, one story with an S-coordinated target, and a story not containing coordinations.

Design. The experimental and filler stories, 96 items in total, were divided into four blocks of 24 stories each. There were two factors in this experiment: (1) ambiguity (of the target sentence), with the levels ambiguous (without comma) and control (with comma), and (2) context type, with the levels bias (duplex topic) and neutral. Each experimental item appeared in the four conditions created by crossing these factors. Ambiguity and context type are therefore within-item factors. Four experimental lists were created using a Latin Square, with equal numbers of items occurring in each condition on each list, and with no list containing more than one version of a given item. The order in which experimental and filler items appeared in each list was determined semirandomly (i.e., allowing maximally two experimental items in consecutive order, but never in the same condition) and was the same for all four lists. Each list was presented to an equal number of participants and each participant only saw one list.

Procedure. Participants read the stories segment-by-segment, in a noncumulative moving window fashion (Just, Carpenter, \& Woolley, 1982). The texts were segmented so as to have no more than 3 words and no more than 25 characters in each segment. Care was taken to have segments correspond to syntactic constituents (e.g., NPs, PPs) and not to mix, for example, verbs and NPs. Each story was preceded by an asterisk on the left-hand side of the screen, indicating where the story would begin (i.e., the position of the first segment of the lead-in sentence). When the button was pushed, the asterisk disappeared and the first segment of the lead-in

other did not. In both experiments a significant garden-path effect was found, which was slightly larger (i.e., $11 \mathrm{~ms}$ ) in the experiment containing content questions. Apart from that, the results from both experiments were virtually identical. This finding that the reading behavior of the participants in these experiments is influenced only to a small extent by the presence of comprehension questions suggests that it is possible to study reading behavior without adding a second task. 
TABLE 4

Sample Experimental Items Used in Experiments 2 and 3, with English Translation in Apostrophes

Lead-in sentence

4.1 De nieuwe collectie avondjurken, die op die avond gepresenteerd werd, sloeg in als een bom in de Parijse modewereld.

'The new collection of evening dresses that was presented that night, really struck home in the fashion world of Paris.'

Neutral context ${ }^{a}$

4.2 Het was dan ook niet vreemd dat het feest naderhand bijzonder uitbundig was.

'It was therefore not surprising that the party afterwards was exhilarating.'

Biasing context ${ }^{a}$

4.3 Toen ze na afloop de modeontwerper op de party ontmoetten, waren de mannequin en de fotograaf dan ook heel enthousiast.

'When they met the fashion designer at the party afterward, the model and the photographer were very enthusiastic.'

Target sentence

4.4 De mannequin omhelsde de ontwerper(,) en de fotograaf opende lachend een fles champagne.

'The model embraced the designer(,) and the photographer opened smilingly a bottle of champagne.'

Exit sentence

4.5 Het werd een onvergetelijke avond.

'This surely was a memorable evening.'

${ }^{a}$ In the experimental materials, either the neutral or the biasing context-sentence was presented. The comma in parentheses in the target-sentence indicates the position of the comma in the control condition.

part of the story appeared; for the rest of the segments each character (including all punctuation marks except the full stop) was replaced by a hyphen. The next button-press revealed the next segment of the sentence, while changing the characters of the first segment to hyphens, and so forth. For practical reasons related to the planned replication using eye tracking (such as the concern that the occurrence of blinks will increase when texts are presented as compared to single sentences; see also the procedure section of Experiment 3), all stories were presented in two parts: the lead-in sentence was presented first, and after a 350-ms delay, the rest of the story was shown. The critical region of the target-sentence, consisting of the disambiguating verb, the postverbal adverb, and an object NP, always appeared at the end of a line, while the subsequent prepositional phrase appeared on the next line. All target-sentences were segmented as shown in example (10) (translated literally from Dutch).

TABLE 5

Sample Filler Materials Used in Experiments 2 and 3 (English Translations)

Neutral Filler

Lead-in sentence

Critical context

Target sentence

Exit sentence

Biasing filler

Lead-in sentence

Critical context

Target sentence

Exit sentence
The old steam-locomotive had been restored by an association of train fanatics.

When the train was to ride again after so many years, a lot of people had gathered to witness the event. The little boy admired the conductor and the engine-driver because they wore such impressive uniforms.

With his mouth wide open he looked at the shining locomotive which puffed out large clouds of steam.

Fashion house Antje had provided the dress for the wedding ceremony that was taking place in the town hall.

When they saw the beautiful dress the bride was wearing, the civil servant and the groom were very impressed.

The civil servant congratulated the bride and the groom while the guests cheered.

It was the start of a wonderful day. 
The model / embraced / the designer / and / the photographer / opened / smilingly /

a bottle / of champagne./

Before the experiment started, participants were given written instructions. In the instructions it was stressed that each story should be read carefully and at a normal speed. In order to "explain" the stories being presented in two parts, and also to keep participants from focusing on the syntactic and topic-structure aspects of the materials, participants were told that the goal of the experiment was to determine how people process the relation between a lead-in sentence and the following story. Note that this lead-in sentence never contained any syntactic or topic-structure cues. After a practice session consisting of three stories, the experiment started. The experiment took 30 to $45 \mathrm{~min}$.

\section{Results}

First, the reading time data were screened for errors and outliers. All reading times less than $50 \mathrm{~ms}$ and greater than $4000 \mathrm{~ms}$ were excluded. After this preliminary screening, all observations were excluded which deviated more than 2 $S D$ s from both the participant and the item means of each segment in each condition; $1 \%$ of the original observations were removed by these procedures.

For each segment in the target sentence two sets of analyses were performed. In one analysis participants were treated as a random factor, and an $F_{1}$-ANOVA was conducted on the means for each participant. In the other analysis, the items were treated as the random factor and an $F_{2^{-}}$ ANOVA was conducted on the means for each item. The factors ambiguity (ambiguous vs control) and context type (bias vs neutral) were treated as within-participants and within-items factors.

In our discussion of the results we will primarily focus on three regions, the disambiguating verb (opened) and the two postdisambiguation regions (smilingly/a bottle). Mean reading times per segment are displayed in Table 6 and are presented graphically in Fig. 1.

1. Disambiguating verb. At the disambiguating verb opened, there was no significant interaction of ambiguity and context type (both $F \mathrm{~s}<$ 1 ), and neither was there a main effect of ambiguity (both $p$-values $>.11$ ). The factor contexttype, however, had a significant main effect $\left(F_{1}(1,31)=7.06, M S_{e}=1109, p<.05 ;\right.$ $\left.F_{2}(1,47)=5.19, M S_{e}=2059, p<.05\right)$, indicating that disambiguating verbs were read faster when the sentence was embedded in a biasing context (a difference of $15 \mathrm{~ms}$ ).

2. Postdisambiguation regions. At the adverb smilingly, the interaction of ambiguity and context type was significant $\left(F_{1}(1,31)=6.62\right.$, $M S_{e}=501, p<.05 ; F_{2}(1,47)=5.48, M S_{e}=$ $942, p<.05)$. This interaction arose because of a significant effect of ambiguity in the neutral context condition, where ambiguous sentences were read 17 ms more slowly than controls $\left(F_{1}(1,31)=4.69, M S_{e}=937, p<.05\right.$;

TABLE 6

Mean Reading Times per Region (ms) as a Function of Context Type and Ambiguity in Experiment 2

\begin{tabular}{|c|c|c|c|c|}
\hline & \multicolumn{4}{|c|}{ Condition } \\
\hline & \multicolumn{2}{|c|}{ Neutral context } & \multicolumn{2}{|c|}{ Biasing context } \\
\hline & Ambiguous & Control & Ambiguous & Control \\
\hline \multicolumn{5}{|l|}{ Region } \\
\hline Object NP & 414 & 443 & 424 & 433 \\
\hline Connective & 385 & 405 & 384 & 391 \\
\hline Ambiguous NP & 413 & 401 & 404 & 389 \\
\hline Disambiguating verb & 413 & 399 & 393 & 388 \\
\hline \multicolumn{5}{|c|}{ Postdisambiguation regions } \\
\hline Adverb & 394 & 377 & 377 & 381 \\
\hline Postadverbial NP & 450 & 452 & 453 & 452 \\
\hline Final word & 639 & 646 & 662 & 629 \\
\hline
\end{tabular}




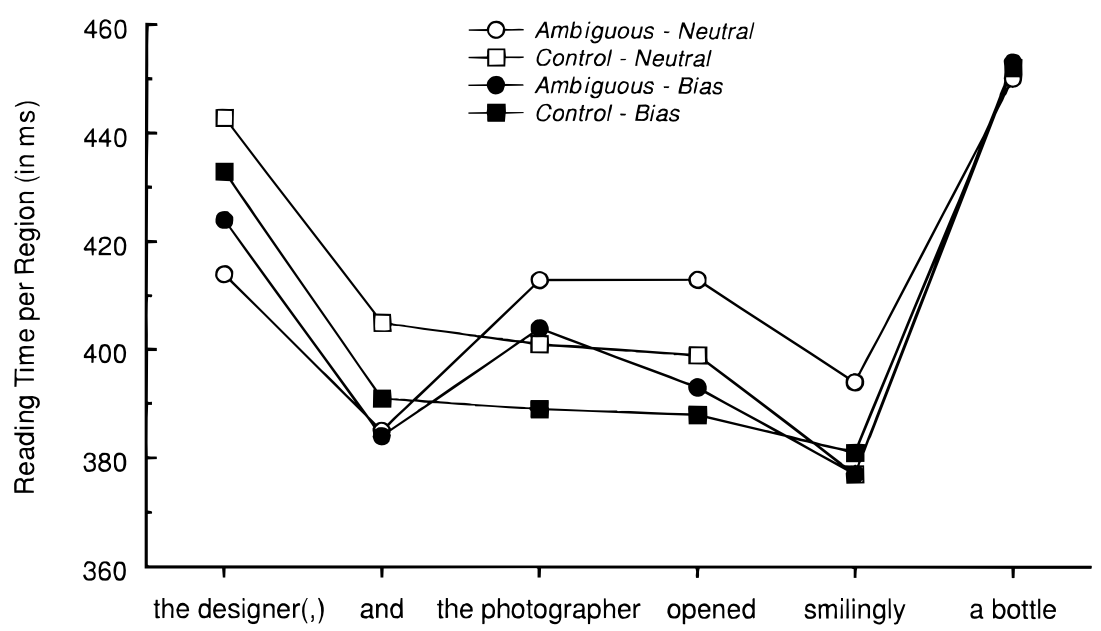

FIG. 1. Mean reading times per region (ms), as a function of ambiguity and context type in Experiment 2. Neutral $=$ neutral context condition; Bias $=$ biasing context condition .

$\left.F_{2}(1,47)=6.63, M S_{e}=1066, p<.05\right)$, while there was no ambiguity effect in the biasing context condition (a 4-ms difference in the other direction; both $F \mathrm{~s}<1$ ). There were no significant main effects. On the subsequent NP a bottle, there was no significant interaction, nor were there significant main effects (all $F$ s $<1)$.

3. Other regions. At the object-NP the designer, where a comma was present in the control sentences, no significant interaction of context type and ambiguity emerged (both $p$-values $>.20$ ), nor was there a main effect of context-type (both $F \mathrm{~s}<1$ ). Object NPs were read $19 \mathrm{~ms}$ more quickly in ambiguous than in control sentences, which was significant in the analysis by items and marginally significant in the analysis by participants $\left(F_{1}(1,31)=3.49\right.$, $M S_{e}=3248, p=.07 ; F_{2}(1,47)=7.19, M S_{e}=$ $2358, p<.05)$. The same pattern of results was found for the reading time pattern at the connective, where there was no significant interaction of context type and ambiguity (both $p$-values $>.20$ ), and no main effect of context type (both $p$-values $>.10$ ). The effect of ambiguity was marginally significant in the analysis by participants but was significant in the itemanalysis $\left(F_{1}(1,31)=3.11, M S_{e}=1982, p=\right.$ $\left..09 ; F_{2}(1,47)=8.20, M S \mathrm{e}=1254, p<.01\right)$, with longer reading times (14 ms) for the connective in the control sentences.

At the ambiguous NP the photographer, there was no significant interaction of ambiguity and context-type. The main effect of ambiguity was significant in the item-analysis and marginally significant by participants $\left(F_{1}(1,31)=2.82\right.$, $M S_{e}=2004, p=.10 ; F_{2}(1,47)=6.44, M S_{e}=$ 1407, $p<.05$ ): Ambiguous NPs were read 14 ms more slowly in ambiguous than in control sentences. The main effect of context type (ambiguous NPs in a biasing context are read $10 \mathrm{~ms}$ faster than in a neutral one) almost reached significance $\left(F_{1}(1,31)=4.09, M S_{e}=926, p=.05\right.$; $\left.F_{2}(1,47)=3.48, M S_{e}=1444, p=.07\right)$. No significant effects were found at the sentence-final region of champagne (all $p$-values $>.10$ ).

\section{Discussion}

The interpretation of the results from this experiment is straightforward. We found clear evidence for processing difficulty in temporarily ambiguous S-coordinations embedded in neutral contexts. Reading times for the first postdisambiguation region (i.e., the adverb) were $17 \mathrm{~ms}$ longer in the ambiguous condition as compared to the unambiguous control condition. More importantly, we found that the topic manipulation in the biasing contexts eliminated this process- 
ing difficulty. This provides strong support for our analysis of the NP-coordination preference in terms of topic-structure simplicity.

The fact that the crucial interaction between ambiguity and context type was found one word downstream from the disambiguating verb (i.e., the adverb) comes as no real surprise, since this "delayed" effect is a common finding in the sentence processing literature with regard to self-paced reading experiments. Though the numerical pattern is already present at the disambiguating verb (i.e., a 14-ms difference in the neutral condition compared to a 5-ms difference in the biasing condition), it only reaches significance one word later.

One notable aspect of these data is that the reading times for the ambiguous sentences in the biasing context barely differ from those for the control sentences in the neutral condition (i.e., from the object NP until the postadverbial NP). This pattern of results is most likely the result of the object NP and the ambiguous NP of the target sentence having already been introduced in the biasing context but not in the neutral context condition. It is less likely that the same thing is happening at the disambiguating verb and the postdisambiguating regions, which have not been mentioned earlier. There, the absence of reading time differences (i.e., between the ambiguous sentence in the biasing context and the control sentence in the neutral context) seems to show that the topic-structure manipulation is as effective as an explicit syntactic signal such as the comma in disambiguating temporarily ambiguous sentences.

Finally, there is the effect of ambiguity at the ambiguous NP, where reading times were some $14 \mathrm{~ms}$ longer for ambiguous NPs in ambiguous sentences than in unambiguous controls. The size of the ambiguity effect was approximately the same for both neutral and biasing contexts. One explanation would be that, since VP-coordination is the preferred continuation at the connective (see Experiment 1, substudies 1a and 1c), readers, expecting a finite verb, will experience processing difficulty when encountering an NP instead. This would explain the effect being present in both context conditions. However, the same must then be predicted to happen in the control sentences (containing a disambiguating comma), since the presence of the comma does not in any way preclude a VP-continuation either (at least not in Dutch). So if the effect were present in both ambiguous and control sentences, we would not have observed any reliable effect of ambiguity. We will return to this issue in the discussion of Experiment 3.

Summarizing, the topic-structure manipulation in the context successfully eliminated the processing difficulty associated with reading temporarily ambiguous S-coordinations, which was present when the same S-coordinations were embedded in neutral contexts. The next experiment will show that the principal results from Experiment 2 can be replicated with eye tracking, indicating that these findings are robust and not attributable to the use of a specific experimental method.

\section{EXPERIMENT 3}

Experiment 3 is a replication of the selfpaced reading experiment discussed in the previous section. In the present experiment eye tracking was used, since this technique permits normal, uninterrupted reading, while at the same time providing a more time-sensitive measure of processing than self-paced reading. This is important because we are interested in finding the earliest point in the sentence where the processing difficulty in the neutral context condition becomes manifest. Recall that in Experiment 2 , the crucial interaction of ambiguity and context-type was found not on the disambiguating verb but one word later.

\section{Method}

Participants. The participants were 32 undergraduate students from the University of $\mathrm{Ni}$ jmegen, who were paid for participation. All had normal, uncorrected vision.

Materials and design. The materials of Experiment 2 were used, with some minor changes. ${ }^{2}$

\footnotetext{
${ }^{2}$ The wording of some of the stories was changed slightly to facilitate reading by replacing some rather low-frequency words and by altering some formulations. We did not make any changes in the critical region of the experimental sentences, i.e., from the disambiguating verb onward.
} 
One line on the screen corresponded to maximally 80 characters. Characters appeared in Courier New, size 12. All experimental sentences had at least three words following the disambiguating word on the same line. The same design was used as in Experiment 2.

Apparatus. Stimuli were presented on an NEC MultiSync 5FG computer monitor. Viewing distance was $85 \mathrm{~cm}$, making $1^{\circ}$ of visual angle equivalent to 4.4 character positions. Eye movements were recorded using an AmTech ET3 eye tracker (Katz, Mueller, \& Helmle, 1987). Both $X$ and $Y$ positions were collected with a sample frequency of $200 \mathrm{~Hz}$ and a spatial resolution of $0.25^{\circ}$. Only the movements of the right eye were recorded. Head movements were minimized by the use of a bite-bar, combined with a chin and forehead rest.

Procedure. Participants were tested individually in a session of approximately $1.5 \mathrm{~h}$. At the start of the session it was verified that participants indeed had normal vision, and a bite-bar was prepared for each individual participant. After a short instruction concerning calibration, the eye tracker was adjusted to the participant and the calibration routine was practiced. Then, the participant received the instructions for the experiment. It was stressed that it was important to read the stories carefully and at normal speed.

The experiment consisted of four blocks of 24 stories each, with a preceding practice session of three stories. After two blocks, the participant was invited to take a short break. All blocks were preceded by a calibration routine, which was inspected off-line. When this calibration was deemed satisfactory, the experiment proceeded. Stories were presented in two parts. First, the lead-in sentence was shown, preceded by a screen with an asterisk, indicating the exact location of the beginning of this sentence. When the participant pushed the button after having finished reading the lead-in sentence, the sentence disappeared from the screen, and the rest of the story was shown. This second part was presented at exactly that location on the screen where the reader would normally continue reading (i.e., beginning at the leftmost position of the line beneath the lead-in sentence), just as in Experiment 2. As was noted in the Procedure of
Experiment 2, the main reason to divide the stories into two parts was a concern about blinkartefacts in the eye-tracking signal. In order to minimize the occurrence of blinks during the important parts of the story (i.e., the critical context sentence and the target sentence), participants were asked not to blink during the second part of the story; blinking was allowed at the asterisk or during the lead-in sentence.

Each story was preceded by a short recalibration routine, by means of which the data could be corrected for possible shifts in the participant's head position.

\section{Results}

For analysis purposes, all target sentences were divided into regions of one or more words, as in (11). These analysis regions were identical to the segments described in Experiment 2.

The model / embraced / the designer / and / the photographer / opened / smilingly / a bottle I of champagne. I

Only the italicized regions were analyzed. ${ }^{3}$ For every region three dependent measures were calculated: first-pass regressions (hereafter, regressions), first-pass reading time, and regression-path duration (RPD). Regressions are defined as regressive eye movements originating from a particular region when visiting that region for the first time, provided that that region was not skipped on an earlier pass through the sentence. Regression percentages are based on the number of times a region was actually fixated in first-pass reading. The first-pass reading time is the time spent in a region before leaving that region to the right or to the left, provided that the reader enters that region for the first time, and that the region was not skipped on an earlier pass through the sentence. And finally, regression-path duration was computed as the time spent in a region in the first pass before leaving that region to the right, plus all the time

\footnotetext{
${ }^{3}$ Since reading time measures pertaining to the connective en ('and') itself were unstable because it was skipped more than $83 \%$ of the time, we performed additional analyses in which it was added to either the preceding region (object NP) or the following region (ambiguous NP). The results of both analyses did not differ from what will be reported below.
} 
spent in regressing to earlier parts of the sentence (see also Brysbaert \& Mitchell, 1996; Konieczny, Hemforth, Scheepers, \& Strube, 1997; Liversedge, Paterson, \& Underwood, 1997; Traxler, Pickering, \& Clifton, 1998).

In the computation of first-pass reading time and RPD, the duration of the saccades (i.e., between the fixations which contributed to these measures) was included. In other words, "time spent" was taken as a variable, instead of "sum of fixation durations," since it is rather implausible that lexical and supralexical processing stops during saccades (cf. Cozijn, 2000; Irwin, 1998; see also Rayner, 1998).

All observations were excluded which deviated more than $3 S D$ s from both the participant and the item means of each region in each condition. Approximately $1 \%$ of the original observations were removed. The subsequent statistical analyses were the same as those described in Experiment 2. Means for all measures are given in Table 7. The results for first-pass reading times are presented graphically in Fig. 2.

In discussing the results for each region, we will concentrate on the findings with respect to first-pass reading time. After that, the results for regressions and regression-path duration will be discussed in a more global way.

\section{First-Pass Reading Time}

1. Disambiguating verb. At the disambiguating verb, a significant main effect of context type $\left(F_{1}(1,31)=4.96, M S_{e}=1012, p<.05\right.$; $\left.F_{2}(1,47)=4.33, M S_{e}=1998, p<.05\right)$ emerged, as well as a marginally significant main effect of ambiguity $\left(F_{1}(1,31)=3.13\right.$, $M S_{e}=1498, p=.09 ; F_{2}(1,47)=3.33, M S_{e}=$ $2403, p=.07)$. However, these main effects were further qualified by the presence of a significant interaction between ambiguity and context-type $\left(F_{1}(1,31)=5.15, M S_{e}=738\right.$, $p<.05 ; F_{2}(1,47)=4.74, M S_{e}=2174, p<$

TABLE 7

Means of First-Pass Reading Time (ms), First-Pass Regressions (Percentages), and Regression-Path Duration (ms) per Region, as a Function of Context Type and Ambiguity in Experiment 3

\begin{tabular}{|c|c|c|c|c|c|}
\hline \multirow{3}{*}{ Measure } & \multirow{3}{*}{ Region } & \multicolumn{4}{|c|}{ Condition } \\
\hline & & \multicolumn{2}{|c|}{ Neutral context } & \multicolumn{2}{|c|}{ Biasing context } \\
\hline & & Ambiguous & Control & Ambiguous & Control \\
\hline \multirow[t]{7}{*}{ First-pass reading time } & Object NP & 292 & 297 & 282 & 282 \\
\hline & Ambiguous NP & 336 & 340 & 313 & 326 \\
\hline & Disambiguating verb & 294 & 271 & 271 & 270 \\
\hline & Postdisambiguation regions & & & & \\
\hline & Adverb & 286 & 279 & 268 & 268 \\
\hline & Postadverbial NP & 266 & 276 & 269 & 258 \\
\hline & Final region & 496 & 494 & 493 & 494 \\
\hline \multirow[t]{7}{*}{ First-pass regressions } & Object NP & 11 & 15 & 10 & 13 \\
\hline & Ambiguous NP & 8 & 8 & 9 & 4 \\
\hline & Disambiguating verb & 6 & 7 & 6 & 6 \\
\hline & Postdisambiguation regions & & & & \\
\hline & Adverb & 11 & 8 & 9 & 8 \\
\hline & Postadverbial NP & 12 & 12 & 9 & 9 \\
\hline & Final region & 20 & 23 & 21 & 22 \\
\hline \multirow[t]{7}{*}{ Regression-path duration } & Object NP & 342 & 383 & 337 & 355 \\
\hline & Ambiguous NP & 377 & 382 & 345 & 348 \\
\hline & Disambiguating verb & 325 & 305 & 300 & 290 \\
\hline & Postdisambiguation regions & & & & \\
\hline & Adverb & 346 & 321 & 310 & 309 \\
\hline & Postadverbial NP & 347 & 334 & 318 & 301 \\
\hline & Final region & 664 & 646 & 674 & 651 \\
\hline
\end{tabular}




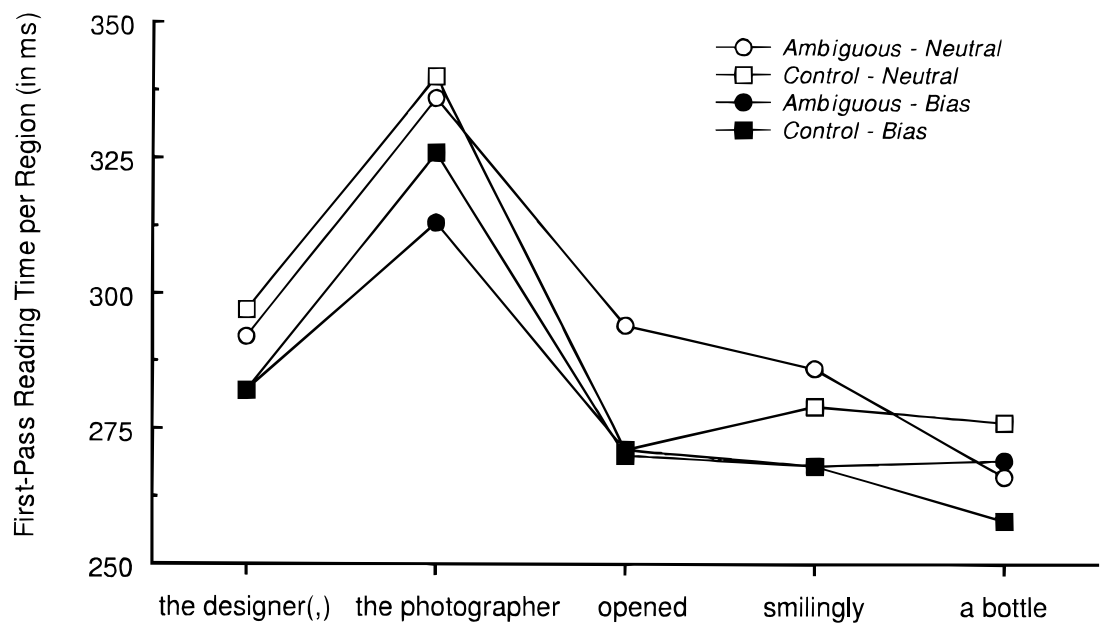

FIG. 2. Means of first-pass reading times per region (ms), as a function of ambiguity and context type in Experiment 3 . Neutral $=$ neutral context condition; Bias $=$ biasing context condition.

$.05)$. In the neutral context condition, first-pass reading time at the verb was significantly longer $(23 \mathrm{~ms})$ for ambiguous sentences than for control sentences $\left(F_{1}(1,31)=6.50, M S_{e}=\right.$ $1303, p<.05 ; F_{2}(1,47)=7.31, M S_{e}=2493$, $p<.05)$. No such effect (1 ms difference) emerged in the biasing context condition (both $F \mathrm{~s}<1)$.

2. Postdisambiguation regions. At the postverbal adverb smilingly, only the main effect of context type was significant: First-pass reading time on the adverb was $15 \mathrm{~ms}$ longer in neutral contexts than in biasing ones $\left(F_{1}(1,31)=6.40\right.$, $M S_{e}=1009, p<.05 ; F_{2}(1,47)=4.80, M S_{e}=$ $1568, p<.05)$. There were no other significant effects (all $F \mathrm{~s}<1$ ). At the next region, a bottle, there were no significant effects at all (all $p$-values $>.14)$.

3. Other regions. At the object NP the designer, the effect of context type was significant by participants and marginally significant by items $\left(F_{1}(1,31)=5.12, M S_{e}=1002, p<.05\right.$; $\left.F_{2}(1,47)=2.86, M S_{e}=2013, p=.10\right)$, indicating a trend for object NPs to be read faster (13 $\mathrm{ms}$ ) in biasing contexts than in neutral contexts. The main effect of ambiguity was not significant, nor was there a significant interaction (all $\left.F_{\mathrm{s}}<1\right)$. There was a marginally significant main effect of context type on the ambiguous $\mathrm{NP}$ the photographer: First-pass reading time tended to be longer for ambiguous NPs in neutral contexts than in biasing contexts. This difference $(18 \mathrm{~ms})$ was significant by participants and almost significant by items $\left(F_{1}(1,31)=\right.$ 8.05, $M S_{e}=1335, p<.01 ; F_{2}(1,47)=3.75$, $\left.M S_{e}=3101, p=.06\right)$. There were no other effects (all $p$-values $>.15$ ). At the sentence-final region of champagne no significant effects were found (all $F_{\mathrm{s}}<1$ ).

\section{Regressions}

Only a few differences in the first-pass regressions came close to being significant. First, at the object NP, comma-placement seemed to slightly increase the occurrence of regressions. The main effect of ambiguity (a 3\% difference) was significant by participants, but not by items $\left(F_{1}(1,31)=5.23, p<.05 ; F_{2}(1,47)=1.74, p=\right.$ .19). There was no significant main effect of context type, nor was there an interaction of context type and ambiguity. Second, at the ambiguous NP the interaction of ambiguity and context type almost reached significance $\left(F_{1}(1,31)=\right.$ $\left.3.59, p=.07 ; F_{2}(1,47)=3.02, p=.09\right)$, probably reflecting the rather small number of regressions in the control-sentences embedded in a biasing context, as compared to the other three conditions, which also gave rise to a marginally significant main effect of ambiguity $\left(F_{1}(1,31)=\right.$ $\left.3.22, p=.08 ; F_{2}(1,47)=3.39, p=.07\right)$. Fi- 
nally, the marginally significant main effect for context type at the postadverbial NP suggested that readers make slightly more regressions $(2 \%)$ in neutral contexts than in biasing contexts $\left(F_{1}(1,31)=3.44, p=.07 ; F_{2}(1,47)=3.79, p=\right.$ $.06)$. No other effects approached significance.

Regression-path duration. Regression-path duration numerically showed the same pattern of results as first-pass reading time, but the effects were statistically less reliable. No significant interaction between ambiguity and contexttype was found anywhere in terms of regression-path duration. A number of main effects were significant or marginally significant. At the disambiguating verb, the effect of context type (i.e., verbs in neutral contexts showing longer regression path durations than in biasing contexts, a difference of $20 \mathrm{~ms}$ ) was significant by participants and marginally significant by items $\left(F_{1}(1,31)=4.31, M S_{e}=2944, p<.05\right.$; $\left.F_{2}(1,47)=2.79, M S_{e}=6472, p=.10\right)$, while the main ambiguity effect (verbs in ambiguous sentences take $15 \mathrm{~ms}$ longer than controls) was marginally significant by items but not significant by participants $\left(F_{1}(1,31)=1.74, M S_{e}=\right.$ 4027, $p=.20 ; F_{2}(1,47)=3.66, M S_{e}=3205$, $p=.06$ ). As for the postdisambiguation regions, a significant main effect of context type was found at the postverbal adverb (24 ms), indicating longer path durations for adverbs in neutral contexts, as compared to the biasing contexts $\left(F_{1}(1,31)=4.37, M S_{e}=4469, p<.05\right.$; $\left.F_{2}(1,47)=5.37, M S_{e}=4560, p<.05\right)$. The main effect of ambiguity did not reach significance $\left(F_{1}(1,31)=1.53, M S_{e}=3530, p=.23\right.$; $\left.F_{2}(1,47)=2.18, M S_{e}=5692, p=.15\right)$. At the second part of the postdisambiguation region, the main effect of context type failed to reach significance in the analysis by participants, but it was marginally significant in the analysis by items $\left(F_{1}(1,31)=2.64, M S_{e}=11446, p=.11\right.$; $\left.F_{2}(1,47)=3.45, M S_{e}=8733, p=.07\right)$, indicating a trend for longer RPDs (31 ms) in neutral contexts as compared to biasing contexts.

At the object NP, there was a marginally significant effect of ambiguity: RPDs tended to be longer in control sentences than in ambiguous sentences. The 29-ms difference was significant by participants but not by items $\left(F_{1}(1,31)=\right.$
5.73, $M S_{e}=4914, p<.05 ; F_{2}(1,47)=2.64$, $\left.M S_{e}=10804, p=.11\right)$. No other effects were found in the object-NP region (all $p$-values $>$ .12 ). With regard to the ambiguous NP, there was a main effect of context-type, with longer path durations $(33 \mathrm{~ms})$ for neutral contexts, as compared to biasing contexts $\left(F_{1}(1,31)=9.35\right.$, $M S_{e}=3830, p<.01 ; F_{2}(1,47)=6.31, M S_{e}=$ $7388, p<.05)$. No other effects were significant here nor in the sentence-final region (all $F \mathrm{~s}<1$ ).

\section{Discussion}

This eye-tracking experiment replicated the main findings obtained with the self-paced reading method in Experiment 2. First of all, a significant interaction between ambiguity and context-type was found, brought about by the presence of processing difficulty for S-coordinated sentences in neutral contexts and the absence of processing difficulty when these sentences were embedded in biasing contexts. This indicates that the manipulation of topic-structure in the context was very effective, thus supporting the view that the NP-coordination preference is actually a preference for topic-structure simplicity. In contrast to Experiment 2, the crucial interaction (i.e., of ambiguity and context-type) in the present experiment was already significant at the disambiguating verb itself.

Second, in discussing the results of Experiment 2 , we suggested that the biasing context was as effective as the comma in the neutral context in disambiguating the ambiguous target sentence. Experiment 3 replicated this finding. For example, first-pass reading times on the disambiguating verb and postdisambiguation regions of ambiguous sentences following biasing contexts equalled (or were even shorter than) reading times on control sentences in the neutral condition (see Table 7). This same pattern of results was observed for the object NP and the ambiguous NP, most probably because both have already been mentioned in biasing contexts, but not in neutral contexts.

One important difference between Experiments 2 and 3 concerns the reading times for the ambiguous NP. In Experiment 2, reading time for the ambiguous NP was significantly longer in ambiguous sentences, as compared to control 
sentences. There was no significant difference in the eye-tracking experiment, and the numerical difference was in the opposite direction: Firstpass reading times and RPDs on the ambiguous NP were shorter in ambiguous sentences than in control sentences. Since the effect of ambiguity that was present in the self-paced reading experiment disappeared in the eye-tracking version, we are inclined to ascribe the reading time differences that were found in Experiment 2 to the so-called "rebound-effect," described by Hoeks, Vonk, Hagoort, and Brown (submitted manuscript). This rebound effect, which seemed to be present only in self-paced reading experiments, is characterized by an increase in reading times at some point in the sentence where an integration cue, such as a comma, is present (presumably reflecting the time needed by the processor to integrate the available syntactic and semantic information), followed by a distinct decrease in reading times on the next three or four words. Hoeks et al. found that this pattern of reading was present even in unambiguous sentences, which strongly suggests that it does not primarily reflect processes related to ambiguity resolution but instead reflects more basic processes of reading (e.g., memory management) or possibly even task-related processing (e.g., maintaining an optimal rhythm of pushing the button in self-paced reading experiments).

Finally, it is somewhat surprising that regression-path duration, which is generally taken to be a very sensitive measure of processing disruption, was not more illuminating in this experiment. Though the pattern of interaction between ambiguity and context type was present numerically in regression-path durations (e.g., at the postverbal adverb), it did not reach significance. This could, of course, be ascribed to chance, but perhaps making regressions was not, on average, the preferred way of solving processing problems in this group of participants or with this kind of stimulus (i.e., whole stories instead of isolated sentences). Future research might shed some light on this issue.

Summarizing, the eye-tracking experiment reported here showed that the processing difficulty that is present when readers are confronted with temporarily ambiguous S-coordinated sen- tences disappears when these sentences are embedded in biasing contexts. The effectiveness of the topic-structure manipulation in these biasing contexts strongly supports the view that, in processing (temporarily) ambiguous sentences, readers use strategies such as the principle of minimal topic-structure.

\section{GENERAL DISCUSSION AND CONCLUSION}

Three experiments were presented that investigated the role of topic-structure in processing the NP versus S-coordination ambiguity. The off-line completion study (i.e., Experiment 1) showed that language users are sensitive to the manipulation of topic-structure when completing ambiguous sentence fragments. For fragments presented in isolation, S-coordination was clearly not a preferred option $(15 \%$ or less of all completions), but introducing a duplextopic context greatly increased the likelihood of S-coordinated completions (up to $80 \%$ ). Experiments 2 and 3 then showed that this sensitivity to topic-structure regularities could also affect online processing. In both experiments, a significant interaction of context type (neutral vs biasing) and ambiguity (ambiguous vs control) was found: In the eye tracking experiment (i.e., Experiment 3) this interaction occurred at the disambiguating verb itself; in the self-paced reading experiment (i.e., Experiment 2), it was found one word later. The interaction reflected the presence of processing difficulty in temporarily ambiguous sentences embedded in a neutral context, which was absent in sentences preceded by duplex-topic contexts. In other words, when there are no topic-structure cues, as in the neutral context condition, readers are inclined to take the NP-coordination option when faced with the NP/S coordination ambiguity, and they subsequently run into trouble when the sentence turns out to be $\mathrm{S}$-coordinated. This suggests that readers adopt a default, minimal, topic-structure (i.e., with only one topic) in the absence of clear cues and, hence, take the ambigous NP as being part of the comment, instead of as a second topic. They will then experience processing difficulty if the default structure turns out to be wrong, since, in that case, both 
topic-structure and syntactic structure will have to be revised.

Although we think the present data suggest a pragmatic origin of the NP-coordination preference, which will be further discussed below, other accounts cannot be ruled out. For instance, within the garden-path/construal framework, the conjoint-NP preference is argued to be primarily motivated by syntactic considerations. Due to the application of the minimal attachment strategy, the ambiguous NP is conjoined with the preceding object NP. When prompted by contextual or other information, this decision has to be revised. Under this account, the lack of processing difficulty in the biasing context-condition in the current experiment would have to be explained by invoking very rapid revision processes that cannot be reliably detected by the measurement apparatus used. However, another way in which the present findings can be accommodated within the garden-path/construal framework is to conceive of the NP/S ambiguity as being an association ambiguity, instead of an attachment ambiguity. In association ambiguities, the parser does not attach the ambiguous phrase (e.g., guided by minimal attachment strategy) but rather associates it to the existing phrase marker (or, to be more precise, to the current thematic processing domain), permitting nonsyntactic information, such as, in this case, topic-structure information, to decide where the ambiguous phrase should be inserted (Frazier \& Clifton, 1996, 1997). This would explain why there is an NP-coordination preference (i.e., because of the principle of minimal topic-structure), and also why it can be overruled by topicstructure information in the context.

In another vein, proponents of constraintbased models may explain the basic NP-coordination preference as being a consequence of the relatively high frequency with which the connective 'and' is used to coordinate NPs as compared to Ss (or VPs, for that matter), that is, if coarse-grained frequency measures are used. To explain the outcome of the present experiments, the principle of minimal topic-structure should be added to the list of constraints that have to be satisfied. However, since a basic feature of constraint-based models is that ambigu- ity is resolved through competition between alternatives at the point of syntactic ambiguity, there should be some sign of competition in the duplex-topic condition between the factor frequency, arguing for NP-coordination, and the contextual information, which argues for S-coordination.

The present experiments do not provide any evidence for the presence of a competition effect. In Experiment 3, there was no sign of an effect of ambiguity (i.e., ambiguous vs control) at the ambiguous NP that could be interpreted as processing difficulty. Furthermore, though there was an ambiguity effect at the ambiguous NP in Experiment 2, it is much more likely that the reading time difference here is caused by a rebound effect, since the effect was present in the neutral condition too, where no competition is expected. There are two possible explanations of this apparent absence of competition effects under a constraint-based account. First, it could be argued that the processor uses information on both topic-structure and the coarse-grained frequencies to come to a decision. In this case it would be necessary to assume that the contextual bias factor was much stronger than the frequency factor, thus minimizing the amount of competition needed to resolve the ambiguity. Alternatively, if the more fine-grained frequency information were available to the parser, together with topic-structure information, no competition would be necessary, since the frequency factor does not bias toward either NP- or S-coordination if grammatical function is taken into account.

It remains somewhat unclear what explanation constraint-based models have to offer for the pattern of results at the connective itself, where we did not find any evidence for competition processes either. At the connective, NP- and VP-coordination are the preferred options, depending on the frequency measure used. This will not provide any conflict between frequency and topic-structure information in the neutral context condition, since both NP- and VP-coordination are compatible with the expected topic-comment structure. However, the question is why there is no sign of competition in the biasing context condition, where topic-structure 
information biases toward S-coordination. It could be argued that the topic-structure information contained in the biasing context only exerts its influence when the ambiguous NP is read, and not beforehand, since it only predicts a topic-structure, not a syntactic structure. In other words, the duplex-topic context predicts only a description of two entities, each performing a (separate) action, but not the syntactic structure this description will take. Only when the ambiguous NP is read will the processor "recognize" it as a second topic and process it accordingly. In that case, no competition is expected at the connective either.

As we have just argued, both gardenpath/construal theory and constraint-based models can provide an account for the data from the current experiments. But it is obvious that the present findings also fit very well in the theoretical framework proposed by Crain and Steedman (1985; see also Altmann \& Steedman, 1988), who stated that "there may be no such thing as an intrinsically garden-pathing sentence structure, but rather that for a given sentence, certain contexts (possibly including the null context) will induce a garden path effect, while others will not" (Crain \& Steedman, 1985, p. 322, original italics). They propose that, at any given point in a sentence, all possible syntactically supported alternatives are available to the sentence processor. One of these alternatives is then selected on the basis of specific knowledge contained in the mental model readers have constructed of the ongoing discourse. Such an account is perfectly compatible with the present findings, since it allows pragmatic factors such as topic-structure to bring about parsing preferences as well as to cancel them.

Although Crain and Steedman focused on one aspect of the specific knowledge in the mental model, namely, information about particular referents as formulated in their principle of referential success, the current results imply that topic-structure information and the principle of minimal topic-structure are at least as important in making parsing decisions. The identification of a second pragmatic principle that is intimately related to a parsing preference might act as an incentive to look for other such principles or regularities in sentence processing. Thus, the principle of parsimony (i.e., choose the alternative requiring the fewest changes to the existing discourse model), which can be said to subsume both the principle of referential success and the principle of minimal topic-structure, may serve as a heuristic device to uncover other ways in which pragmatic information can exert an influence on sentence comprehension.

In conclusion, the experiments described here presented clear evidence for the importance of topic-structure information in online sentence processing and its immediate influence on ambiguity resolution. Building on the work of Crain and Steedman (1985), this investigation of the role of topic-structure in ambiguity resolution should be considered as another step toward integrating theories of pragmatics and models of sentence processing.

\section{REFERENCES}

Altmann, G. T. M., \& Steedman, M. (1988). Interaction with context during human sentence processing. Cognition, 30, 191-238.

Altmann, G. T. M., Garnham, A., \& Dennis, Y. (1992). Avoiding the garden path: Eye movements in context. Journal of Memory and Language, 31, 685-712.

Britt, M. A. (1994). The interaction of referential ambiguity and argument structure in the parsing of prepositional phrases. Journal of Memory and Language, 33, 251-283.

Brysbaert, M., \& Mitchell, D. C. (1996). Modifier attachment in sentence parsing: Evidence from Dutch. The Quarterly Journal of Experimental Psychology, 49, 664-695.

Clifton, C., Jr., \& Ferreira, F. (1989). Ambiguity in context. Language and Cognitive Processes, 4, SI 77-103.

Cozijn, R. (2000). Integration and inference in understanding causal sentences. Doctoral dissertation, Tilburg University, Tilburg, The Netherlands.

Crain, S., \& Steedman, M. (1985). On not being led up the garden path: The use of context by the psychological syntax processor. In D. Dowty, L. Karttunen, \& A. Zwicky (Eds.), Natural language parsing: Psychological, computational, and theoretical perspectives (pp. 320-358). Cambridge: Cambridge Univ. Press.

Frazier, L. (1987a). Syntactic processing: Evidence from Dutch. Natural Language and Linguistic Theory, 5 , 519-559.

Frazier, L. (1987b). Sentence processing: A tutorial review. In M. Coltheart (Ed.), Attention and performance XII: The psychology of reading (pp. 601-681). Hillsdale, NJ: Erlbaum.

Frazier, L., \& Clifton, C., Jr. (1996). Construal. Cambridge, MA: MIT Press. 
Frazier, L., \& Clifton, C., Jr. (1997). Construal: Overview, motivation, and some new evidence. Journal of Psycholinguistic Research, 26, 277-295.

Geerts, G., Haeseryn, W., de Rooij, J., \& van den Toorn, M. C. (1984). Algemene Nederlandse Spraakkunst [General grammar and usage of Dutch]. Groningen: Wolters-Noordhof.

Irwin, D. E. (1998). Lexical processing during saccadic eye movements. Cognitive Psychology, 36, 1-27.

Just, M. A., Carpenter, P. A., \& Woolley, J. D. (1982). Paradigms and processes in reading comprehension. Journal of Experimental Psychology: General, 111, 228-238.

Katz, B., Mueller, K., \& Helmle, H. (1987). Binocular eye movement recording with CCD arrays. Neuro-ophtalmology, 7, 81-91.

Konieczny, L., Hemforth, B., Scheepers, C., \& Strube, G. (1997). The role of lexical heads in parsing: Evidence from German. Language and Cognitive Processes, 12, 307-348.

Lambrecht, K. (1994). Information structure and sentence form: Topic, focus, and the mental representation of discourse referents. Cambridge, MA: University Press.

Liversedge, S. P., Paterson, K. B., \& Underwood, G. (1997). Exploring the effects of quantifiers on parsing. Poster presented at the Tenth CUNY Conference on Human Sentence Processing, Santa Monica, CA.

MacDonald, M. C., Pearlmutter, N. J., \& Seidenberg, M. S. (1994). Lexical nature of syntactic ambiguity resolution. Psychological Review, 101, 676-703.

Mitchell, D. C., Corley, M. M. B., \& Garnham, A. (1992). Effects of context in human sentence parsing: Evidence against a discourse-based proposal mechanism. Journal of Experimental Psychology: Learning, Memory, and Cognition, 18, 69-88.

Murray, W., \& Liversedge, S. (1994). Referential context and syntactic processing. In C. Clifton, Jr., L. Frazier, \& K. Rayner (Eds.), Perspectives on sentence processing (pp. 359-388). Hillsdale, NJ: Erlbaum.
Ni, W., Crain, S., \& Shankweiler, D. (1996). Sidestepping garden paths: Assessing the contributions of syntax, semantics, and plausibility in resolving ambiguities. Language and Cognitive Processes, 11, 283-334.

Rayner, K. (1998). Eye movements in reading and information processing: 20 years of research. Psychological Bulletin, 3, 372-422.

Rayner, K., Garrod, S., \& Perfetti, C. A. (1992). Discourse influences during parsing are delayed. Cognition, 45, 109-139.

Renkema, J. (1979). Schrijfwijzer: Handboek voor duidelijk taalgebruik. [Guidelines for Writing: Handbook for transparent language use]. The Hague: Staatsuitgeverij.

Spivey, M. J., \& Tanenhaus, M. K. (1998) Syntactic ambiguity resolution in discourse: Modeling the effects of referential context and lexical frequency. Journal of Experimental Psychology: Learning, Memory, and Cognition, 6, 1521-1543.

Tanenhaus, M. K., \& Trueswell, J. C. (1995). Sentence comprehension. In J. L. Miller \& P. D. Eimas (Eds.), Speech, language, and communication (pp. 217-262). San Diego: Academic Press.

Traxler, M. J., Pickering, M. J., \& Clifton, C., Jr. (1998). Adjunct attachment is not a form of lexical ambiguity resolution. Journal of Memory and Language, 39, 558-592.

Van Berkum, J. J. A, Brown, C. M., \& Hagoort, P. (1999). Early referential context effects in sentence processing: Evidence from event-related brain potentials. Journal of Memory and Language, 41, 147-182.

Zagar, D., Pynte, J., \& Rativeau, S. (1997). Evidence for early-closure attachment on first-pass reading times in French. Quarterly Journal of Experimental Psychology, 50A, 421-438.

(Received September 22, 2000)

(Revision received February 8, 2001)

(Published online October 3, 2001) 